\title{
Micromecanizado de materiales cerámicos mediante láser de femtosegundo
}

\author{
P. MORENO*, C. MÉNDEZ, A. GARCíA, I. ARIAS, E. CONEJERO, L. ROSO \\ Grupo de Óptica, Departamento de Física Aplicada, Universidad de Salamanca, 37008 Salamanca \\ *Departamento de Ingeniería Mecánica, Universidad de Salamanca, 37700 Béjar (Salamanca)
}

\begin{abstract}
En este trabajo presentamos la aplicación de pulsos láser ultracortos ( 110 fs @ 1 kHz; hasta $1.1 \mathrm{~mJ} /$ pulso) al micromecanizado de materiales cerámicos, en concreto Rubalit ${ }^{\mathrm{TM}} 708 \mathrm{~S}$, un material compuesto principalmente de alúmina y empleado en la industria microelectrónica. El mecanismo de eliminación de material es la ablación directa, que difiere de la ablación térmica empleada por los láseres convencionales en la prácticamente total ausencia de efectos térmicos, lo que redunda en un aumento significativo de la precisión y calidad del mecanizado. Mediante técnicas basadas en la difracción óptica determinamos el umbral de energía necesario para que tenga lugar el proceso de ablación directa. Con ese dato y regulando la energía por pulso y el número de pulsos somos capaces de producir mecanizados del diámetro y profundidad deseados. Además, el procesado se realiza en aire. Demostramos que es posible ejecutar micromecanizado de calidad mediante este procedimiento en cualquier material cerámico, independientemente de sus propiedades mecánicas, variando ligeramente los parámetros del proceso. En este caso, además de la alúmina hemos procesado SiN sinterizado, un material de uso extendido en la industria.
\end{abstract}

Palabras Clave: Ablación. Láser de femtosegundo. Alúmina.

\section{Femtosecond laser micromachining of ceramics}

In this work, we present the application of ultrashort and intense laser pulses (110 fs @ $1 \mathrm{kHz}$; up to $1.1 \mathrm{~mJ} / \mathrm{pulse}$ ) to the micromachining of ceramics, specifically Rubalit ${ }^{\mathrm{TM}} 708 \mathrm{~S}$, a material based on alumina and widely used as a substrate in microelectronics. The mechanism for removing material is the so called direct ablation. It differs from thermal ablation of conventional lasers in the practically total absence of thermal effects which produces a remarkable increase of quality and precision of the machining. By means of an optical diffraction-based technique we find out the energy density threshold to work in the direct ablation regime. Adjusting the energy per pulse as well as the number of pulses, we are able to drill holes of the desired diameter and depth. In addition, processing is developed in air. We also demonstrate that high quality fs-laser micromachining is suitable for every ceramic, whatever the mechanical properties, with similar working parameters. In order to show this point, we have also processed sintered $\mathrm{SiN}$, a material of wide-ranging interest in industry.

Keywords: Ablation. Femtosecond laser. Alumina.

\section{INTRODUCCIÓN}

Desde el desarrollo de los primeros láseres hace más de cuatro décadas, las aplicaciones de esta herramienta en los más variados campos científicos y tecnológicos han sido innumerables [1]. El espectacular avance de los conocimientos sobre los procesos no lineales en materiales, junto al de la optoelectrónica, ha permitido disponer muy recientemente de fuentes de radiación láser que son capaces de emitir pulsos de muy corta duración y suficiente energía que, convenientemente focalizados, dan lugar a densidades de energía capaces de producir la ablación directa de materiales sólidos tanto metálicos como dieléctricos [2-8]. El mecanismo consiste básicamente en la ionización por procesos no lineales de los átomos o moléculas de la superficie del material irradiado, formándose un plasma denso que se expande al término del pulso -típicamente de duración en torno a los 100 fs- sin tiempo para que se produzca difusión del calor en la estructura sólida -que requiere tiempos del orden de las decenas de ps-, dando lugar a la eliminación del material sin apenas calentamiento [9].

Esto convierte a los láseres ultracortos e intensos en una herramienta muy prometedora a la hora de producir mecanizado de gran precisión y calidad con dimensiones típicas de micras [10]. Las ventajas se pueden resumir en: (a) el mínimo daño térmico y mecánico que se produce en el material adyacente a la zona de procesado, no observándose restos de material fundido y resolidificado, ni grietas o fisuras; (b) la aplicabilidad universal del proceso, ya que se puede emplear para cualquier tipo de material y no se necesitan entornos de trabajo con condiciones específicas; (c) la posibilidad de depositar sobre el material las cantidades deseadas de energía con gran rapidez y precisión, lo que se traduce en una enorme precisión dimensional y calidad geométrica del mecanizado, una elevada reproducibilidad y la ausencia de postprocesado.

El mayor inconveniente de esta tecnología radica en la baja velocidad de proceso -requisito primordial en cualquier aplicación industrial- lo que, de momento, impide la competencia con otros procesos de menor precisión y calidad, como el mecanizado con láseres de pulsos algunos órdenes de magnitud más largos [11]. Asimismo, al no ser una tecnología madura, el coste de inversión en equipos es todavía exageradamente alto en relación a procedimientos ya existentes. Hoy en día asistimos al debate del futuro industrial de esta tecnología, aunque parece claro que ya se empiezan a encontrar 
aplicaciones concretas en entornos industriales relacionados con las micro y nanotecnologías [12].

En particular, ciñéndonos al ámbito del vidrio y los cerámicos, ya se emplea en el micromecanizado de dispositivos en campos como la biomedicina o la bioquímica, en dispositivos ópticos, sensores, MEMS o del tipo lab-on-chip. Sobre este tipo de materiales, la práctica total ausencia de efectos térmicos en la zona adyacente a la zona mecanizada, además de la posibilidad de producir geometrías complejas de gran calidad, es fundamental.

\section{DISPOSITIVO EXPERIMENTAL}

La producción de pulsos capaces de provocar la ablación directa de materiales cerámicos requiere un conjunto de fuentes láser, dispositivos ópticos y equipos de control bastante complejo. El esquema básico de la instalación se muestra en la Fig.1. Muy recientemente han aparecido las primeras máquinas de procesado compactas que utilizan este tipo de láseres [13].

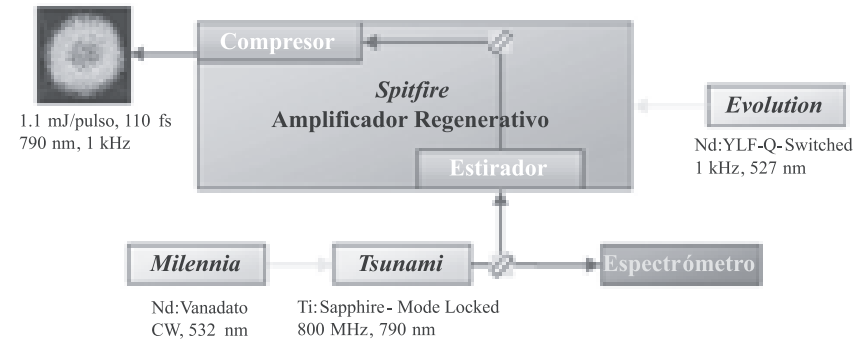

Figura 1: Esquema con los elementos básicos del sistema generador de pulsos ultracortos e intensos.

La fuente principal emplea un medio activo de Ti:Zafiro bombeado ópticamente, que produce pulsos de duración en torno a los $100 \mathrm{fs}$, longitud de onda de $790 \mathrm{~nm}$ y energías del orden de los $10 \mathrm{~nJ}$ por pulso. Para conseguir que esos pulsos adquieran la energía suficiente para producir ablación directa, se hacen pasar por un amplificador regenerativo que emplea la tecnología CPA (Chirped Pulse Amplification) [14] y que logra aumentar la energía de cada pulso sin alterar apreciablemente la duración de los mismos. Empleando esta técnica, nuestro sistema proporciona pulsos de duración en torno a los 110 fs y energías máximas de $1.1 \mathrm{~mJ}$ por pulso con una tasa de repetición de $1 \mathrm{kHz}$. La salida está polarizada linealmente.

La distribución espacial de energía en cada pulso corresponde aproximadamente a un modo $\mathrm{TEM}_{00}$ (gaussiano) cuya sección a la salida del sistema de generación tiene un diámetro de aproximadamente $8.5 \mathrm{~mm}$ (criterio $1 / \mathrm{e}^{2}$ ). Los pulsos se conducen a través del aire a la zona de procesado siendo innecesario el empleo de vacío, atmósferas protectoras o extractores del material removido por las características del mecanismo de ablación directa. Mediante un sistema de espejos a $45^{\circ}$ se dirigen los pulsos en incidencia vertical sobre el material que se desea procesar.

Para conseguir la densidad de energía suficiente para producir la ablación directa del material se focalizan los pulsos mediante un doblete acromático de $100 \mathrm{~mm}$ de distancia focal, situando la superficie del material en el plano focal. Este tipo de óptica de focalización produce las densidades de energía necesarias con una profundidad de foco muy grande que posibilita el mecanizado de espesores hasta de unos $2 \mathrm{~mm}$ permitiendo lograr, por ejemplo, taladros pasantes de alta calidad geométrica.

Las muestras de material se sitúan sobre una base con movimiento micrométrico en los tres ejes, con la posibilidad de incorporar motores para la realización de series de taladros, ranuras $\mathrm{u}$ otras formas geométricas más complejas.

\section{UMBRAL DE ABLACIÓN DIRECTA}

Para desencadenar los procesos no lineales de ionización que conducen a la ablación directa del material, los pulsos láser tienen que cumplir dos requisitos: (1) deben ser lo suficientemente cortos para asegurar una transmisión de energía despreciable al material adyacente a la zona de focalización de los pulsos; (2) deben tener la energía suficiente para que se supere el umbral de ionización multifotónica en los primeros instantes de la interacción con el material.

El primer requisito queda asegurado con los pulsos de 110 fs de los que se dispone, cuya duración es varios órdenes de magnitud inferior a los tiempos típicos de acoplamiento entre electrones y fonones (del orden de decenas o centenas de ps dependiendo de los materiales). El segundo es necesario para que los electrones libres generados por ionización multifotónica sean lo suficientemente numerosos para que, acelerados en el campo electromagnético del láser, provoquen un proceso de ionización en avalancha por colisiones con los átomos que se encuentren en su camino. De esta manera se generará el plasma de densidad crítica que, una vez finalizado el pulso, se expande a gran velocidad debido al mecanismo de explosión coulombiana.

La densidad de energía que se necesita para que tengan lugar los procesos de ionización multifotónica depende, para una longitud de onda dada, de cada material y es lo primero que hay que determinar. Los procesos de absorción e ionización multifotónica son más probables cuanto más grande sea la longitud de onda. Por este motivo, el empleo de longitudes de onda en el infrarrojo es el más indicado.

Para determinar la densidad de energía umbral para la ablación directa $\left(\mathrm{F}_{\mathrm{th}}\right)$ empleamos una técnica basada en la focalización sobre la superficie del material del patrón de difracción del haz láser por una abertura circular de diámetro D inferior al de su sección transversal [15]. En ese caso, la distribución de energía sobre la superficie corresponde a la función de Airy, con un disco central con el máximo de densidad de energía $\left(\mathrm{F}_{0}\right)$ y unos anillos concéntricos de energía decreciente, cuyos radios dependen del diámetro de la apertura interpuesta y de la longitud de onda del láser.

Fijando D y variando las energías de los pulsos, se observa que, a partir de una determinada energía, solamente se elimina material en la zona del disco central. Midiendo los diámetros de las zonas dañadas para las diferentes energías y comparando con la distribución de densidades de energía sobre la superficie para las distintas energías de pulso y el diafragma utilizado, obtenemos $\mathrm{F}_{\text {th }}$.

Este umbral decrece con el número de pulsos debido a efectos de incubación y se estabiliza a partir de un número de pulsos típicamente entre 50 y 100, según los materiales. Como queremos eliminar espesores relativamente grandes de material nos interesa determinar $\mathrm{F}_{\text {th }}$ para un número elevado de pulsos. 


\section{MICROMECANIZADO DE RUBALIT TM708S}

El material procesado es Rubalit ${ }^{\mathrm{TM}} 708 \mathrm{~S}$, un compuesto basado en la alúmina (96\%), empleado en la industria microelectrónica. El material se presenta en forma de obleas de $10 \mathrm{~cm}$ de diámetro con espesor de $250 \mu \mathrm{m}$. Las propiedades de este material son las comunes a la mayoría de los cerámicos avanzados como la refractariedad, baja densidad, dureza y resistencia mecánica a compresión, a las que une un bajo coeficiente de expansión térmica y resistencia al choque térmico, gran resistencia a la corrosión, al ataque químico y al desgaste y baja conductividad eléctrica. Se emplea típicamente como substrato en la fabricación de componentes electrónicos y sensores, como aislante eléctrico y térmico, en prótesis médicas, en componentes antifricción en elementos mecánicos, crisoles, etc.

En la Fig.2 se muestran imágenes de microscopía electrónica de barrido (Zeiss DSM 940) en las que se observa el efecto de irradiar el material con 100 pulsos de energías 1.05, 0.72, $0.42,0.32,0.24$ y $0.18 \mathrm{~mJ}$ respectivamente, interponiendo un diafragma con $\mathrm{D}=3 \mathrm{~mm}$. La medida de los diámetros produce una estimación de $\mathrm{F}_{\text {th }}$ de $1.6 \pm 0.3 \mathrm{~J} / \mathrm{cm}^{2}$ (Fig.3). La determinación de esta densidad umbral para la alúmina no había sido realizada hasta la fecha.

El diámetro de los taladros se controla combinando diámetros del diafragma y energías de los pulsos. Solamente la región del material sobre la que la densidad de energía supere $\mathrm{F}_{\text {th }}$ será eliminada, sin apenas efecto sobre el material adyacente, como se observa en la imagen SEM de la Fig.4, que corresponde a irradiar con 2000 pulsos de $\mathrm{F}_{0}$ igual a $2.5 \mathrm{~J} / \mathrm{cm}^{2}$ con un diafragma de $\mathrm{D}=1.5 \mathrm{~mm}$. El material que aparece sobre las paredes del taladro es material expelido de la superficie irradiada y redepositado, que se podría eliminar simplemente aplicando un flujo de aire a presión.

El empleo de densidades de energía excesivas $\left(\mathrm{F}_{0}>>\mathrm{F}_{\mathrm{th}}\right)$ hace que a la ablación directa se sumen efectos derivados de la absorción de energía por parte del plasma formado, que puede reradiar al material circundante produciendo aumentos de temperatura que provoquen fusión y resolidificación del material, oxidaciones o defectos mecánicos como grietas o fisuras producto de gradientes térmicos, que redundan en una pérdida de calidad del taladro. En la Fig.4 no se aprecia ninguno de estos efectos, típicos de la ablación de tipo térmico y ausentes en la ablación directa.

La profundidad de mecanizado está determinada -una vez fijada $\mathrm{F}_{0}$ - por el número de pulsos utilizado, siendo típicos espesores de la capa removida por pulso entre las decenas de nm y la $\mu \mathrm{m}$ dependiendo de los materiales y de $\mathrm{F}_{0}$ [16]. Para un espesor dado, la forma de los taladros es más o menos cónica, tanto más cuanto menor sea $\mathrm{F}_{0}$ y el número de pulsos empleados. En las Figs. 5a y 5b comparamos los taladros producidos por 2000 pulsos para $\mathrm{F}_{0}$ igual a $5.4 \mathrm{y} 9.9 \mathrm{~J} / \mathrm{cm}^{2}$ respectivamente, mientras que en las Figs. 5 c y $5 \mathrm{~d}, \mathrm{~F}_{0}$ es $5.4 \mathrm{~J} / \mathrm{cm}^{2}$ pero el número de pulsos es de 1000 y 10000 respectivamente. El efecto de conicidad es debido a la disminución de la densidad de energía a medida que nos alejamos del foco y a la reflectividad diferencial de las componentes de la luz linealmente polarizada, cuestiones que esperamos solventar próximamente.

En todos los casos, el procesado se ha realizado en aire. El empleo del vacío en la zona del procesado no es necesario, si bien se ha demostrado que redunda en calidades dimensionales y geométricas superiores [10] al facilitar la eyección del plasma producido.

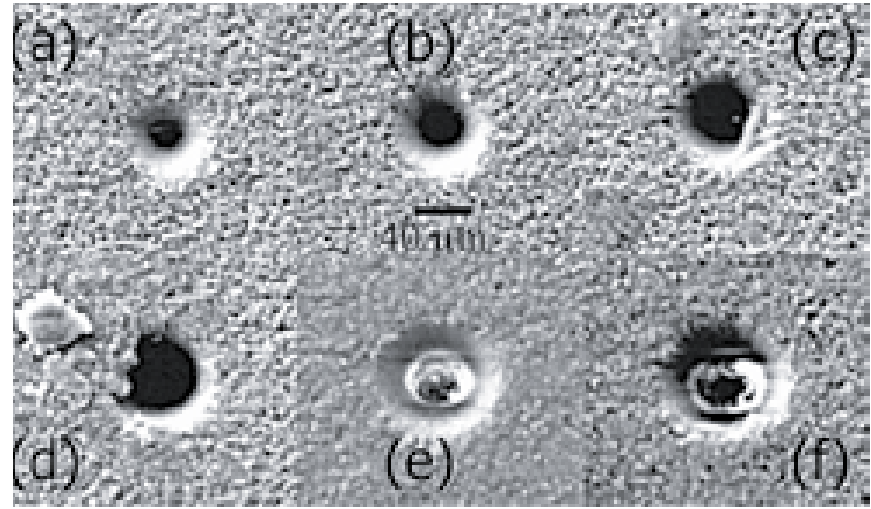

Figura 2: Micrografías SEM mostrando la ablación directa del Rubalit $^{\mathrm{TM}} 708 \mathrm{~S}$ efectuada con 100 pulsos interponiendo un diafragma de $\mathrm{D}=3 \mathrm{~mm}$ y para diferentes energías de los pulsos: (a) 0.18 ; (b) 0.24 ; (c) 0.32 ; (d) 0.42 ; (e) 0.72 y (f) $1.05 \mathrm{~mJ}$.

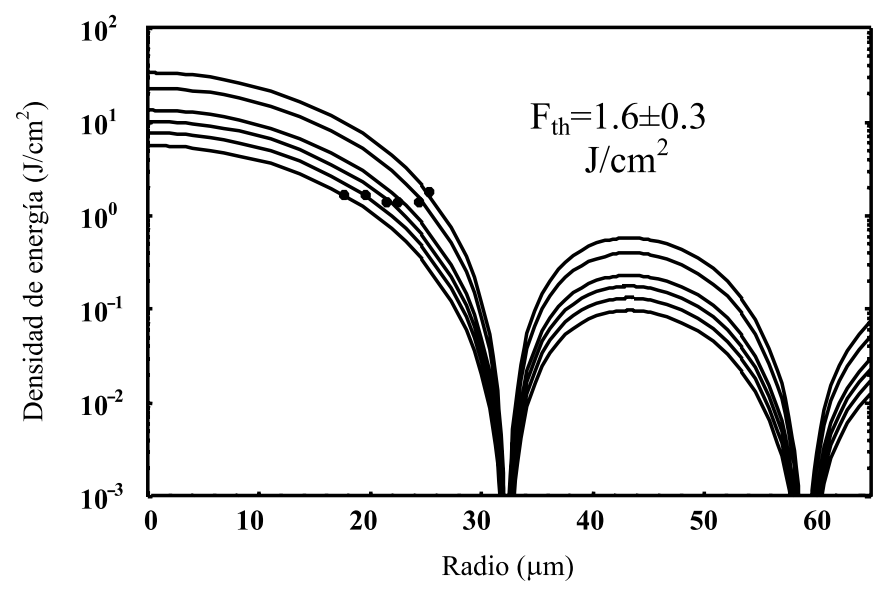

Figura 3: Determinación del umbral de ablación directa del Rubalit ${ }^{\mathrm{TM}} 708 \mathrm{~S}$ para 100 pulsos. Las curvas representan la distribución de energía sobre la superficie para las energías de los pulsos de la Fig.2. Los puntos sobre las curvas indican el radio de la zona dañada para cada una de esas energías.

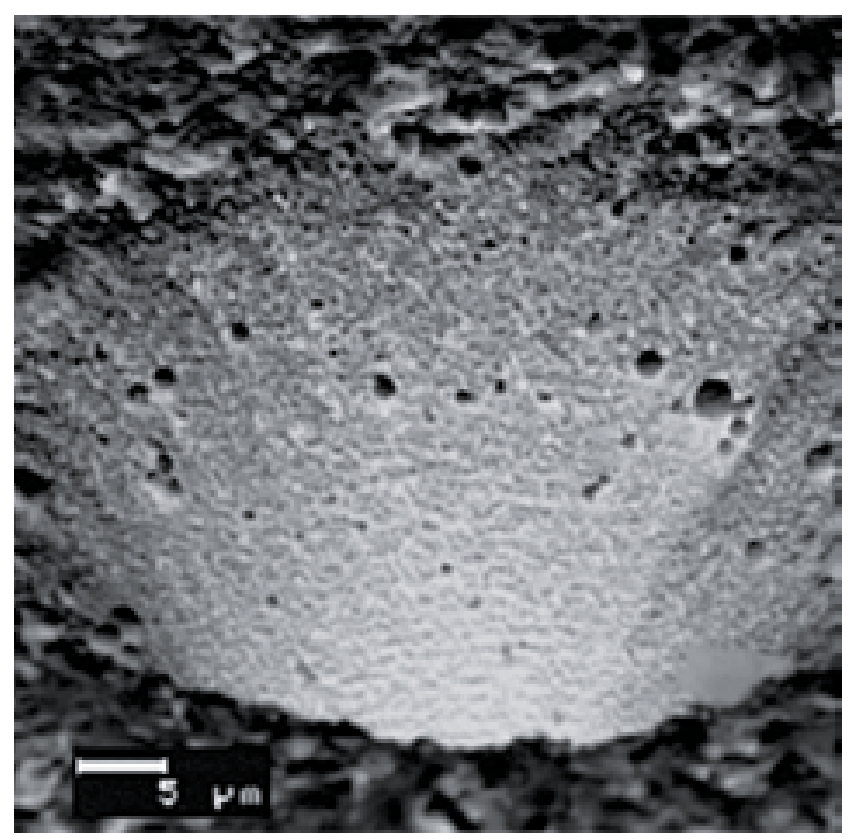

Figura 4: Micrografía SEM de la entrada de un taladro en Rubalit ${ }^{\mathrm{TM}} 708 \mathrm{~S}$ efectuado con 2000 pulsos, interponiendo un diafragma de $\mathrm{D}=1.5 \mathrm{~mm}$ lo que produce una $\mathrm{F}_{0}=2.5 \mathrm{~J} / \mathrm{cm}^{2}$. 


\section{MICROMECANIZADO DE SiN SINTERIZADO}

Por las características del mecanismo de ablación directa, la remoción de material mediante este procedimiento puede ser llevada a cabo en cualquier otro material cerámico sin modificar el dispositivo experimental, solamente regulando la energía de los pulsos para irradiar las superficies con una $\mathrm{F}_{0}$ ligeramente superior a la $\mathrm{F}_{\mathrm{th}}$ del material a procesar.

Como ejemplo presentamos taladros efectuados por el mismo procedimiento en una muestra de SiN sinterizado, material cuyas aplicaciones incluyen el recubrimiento de plaquitas de mecanizado o pistas de rodamiento, las cubiertas de termopares, etc. En este caso, la determinación de $\mathrm{F}_{\text {th }}$ resultó en un valor de $0.25 \pm 0.05 \mathrm{~J} / \mathrm{cm}^{2}$, notablemente inferior al de la alúmina, lo que supone poder emplear pulsos menos energéticos. En la Fig.6 se muestran imágenes de microscopía óptica (Leica DMILM) de mecanizados efectuados sobre este material con $\mathrm{F}_{0}$ igual a 1.7 y $2.2 \mathrm{~J} / \mathrm{cm}^{2}$ respectivamente. Las características de la zona mecanizada son similares a las obtenidas para el Rubalit ${ }^{\mathrm{TM}} 708 \mathrm{~S}$.

\section{CONCLUSIONES}

En este trabajo hemos mostrado la eficacia del empleo de pulsos láser ultracortos e intensos para conseguir micromecanizado de materiales cerámicos con gran precisión y calidad geométrica, evitando los inconvenientes que supone el calentamiento del material en otras técnicas de micromecanizado.

Centrándonos en un material basado en la alúmina y de gran interés en la industria de microprocesadores hemos establecido los parámetros de proceso convenientes para producir la eliminación de material mediante el mecanismo de ablación directa y hemos determinado el efecto que sobre las estructuras mecanizadas tienen variables como la energía y el número de los pulsos empleados.

Hemos mostrado la versatilidad del procedimiento, pues esta tecnología no requiere de unas condiciones especiales para el procesado, realizándose directamente en aire. Además, la técnica es extensible al micromecanizado de otros materiales cerámicos, en este caso $\mathrm{SiN}$, suponiendo únicamente pequeñas modificaciones en los parámetros de proceso y obteniéndose calidades similares a las obtenidas en la alúmina.

\section{AGRADECIMIENTOS}

Agradecemos la financiación de este trabajo al Ministerio de Ciencia y Tecnología de España (proyecto BFM200200033) y a la Junta de Castilla y León (proyecto SA107/03). El Rubalit ${ }^{\mathrm{TM}} 708 \mathrm{~S}$ fue suministrado por el Centro Nacional de Microelectrónica (CSIC) de Barcelona, al que agradecemos su cooperación. Las micrografías SEM han sido obtenidas en el Servicio de Microscopía Electrónica de la Universidad de Salamanca.

\section{BIBLIOGRAFÍA}

1. W. Steen: "Laser Material Processing". Springer Verlag, London (Reino Unido) 1999.

2. P.P. Pronko, S.K.Dutta, J. Squier, J.V. Rudd, D. Du, G. Mourou: "Machining of sub-micron holes using a femtosecond laser at $800 \mathrm{~nm}^{\prime \prime}$. Opt. Commun. 114, 106-110 (1995)

3. C. Momma, B.N. Chichkov, S. Nolte, F. von Alvensleben, A. Tünnermann, H. Welling "Short-pulse laser ablation of solid targets". Opt. Commun. 129, 134-142 (1996)

4. B.N. Chichkov, C. Momma, S. Nolte, F. von Alvensleben, A. Tünnermann: "Femtosecond, picosecond and nanosecond laser ablation of solids". Appl. Phys. A 63, 109-115 (1996)

5. B.C. Stuart, M.D. Feit, S. Herman, A.M. Rubenchik, B.W. Shore, M.D. Perry: "Optical ablation by high power short pulse lasers". J. Opt. Soc. Am. B 13, 459-468 (1996)

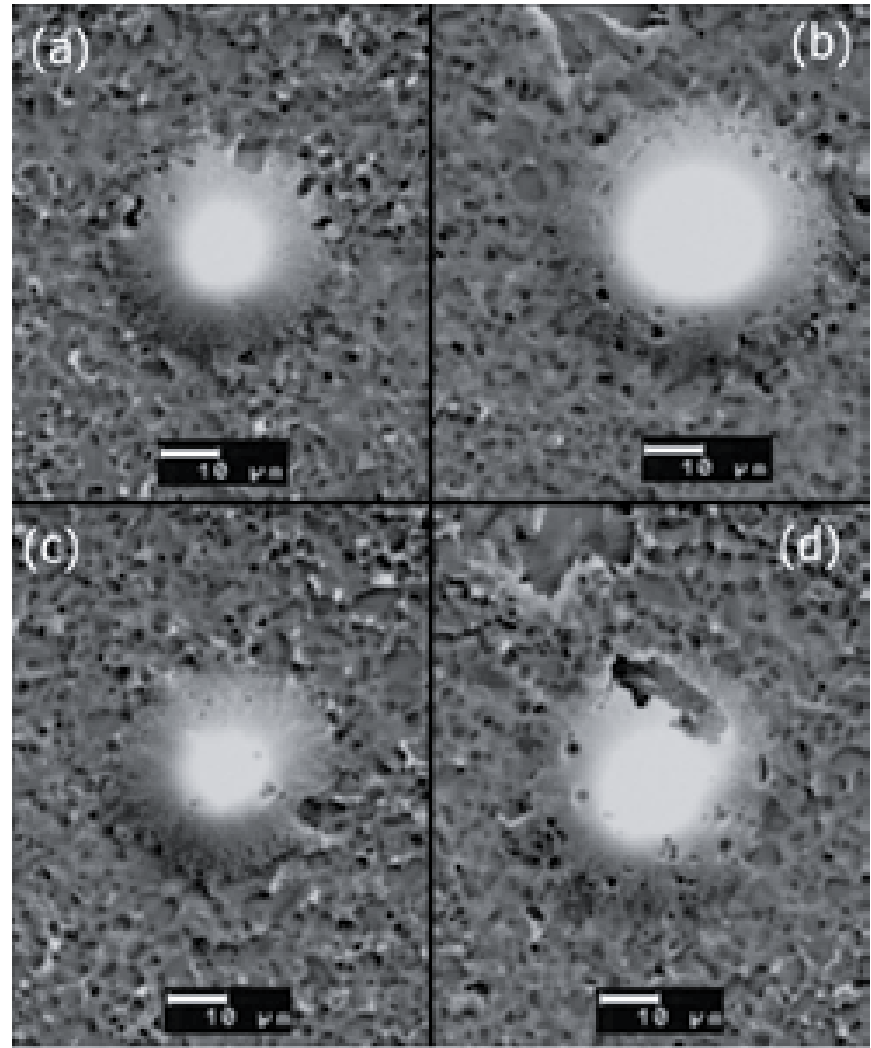

Figura 5: Micrografías SEM de taladros efectuados con los siguientes parámetros: (a) 2000 pulsos y $\mathrm{F}_{0}=5.4 \mathrm{~J} / \mathrm{cm}^{2}$; (b) 2000 pulsos y $\mathrm{F}_{0}=9.9$ $\mathrm{J} / \mathrm{cm}^{2}$; (c) 1000 pulsos y $\mathrm{F}_{0}=5.4 \mathrm{~J} / \mathrm{cm}^{2}$; (d) 10000 pulsos y $\mathrm{F}_{0}=5.4 \mathrm{~J} / \mathrm{cm}^{2}$.

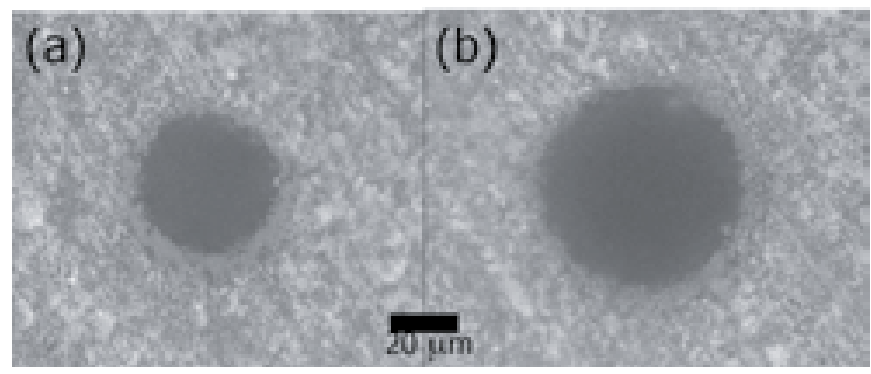

Figura 6: Imágenes de microscopio óptico de taladros efectuados en SiN con los siguientes parámetros: (a) 2000 pulsos, $\mathrm{D}=3 \mathrm{~mm}$ y $\mathrm{F}_{0}=1.7$ $\mathrm{J} / \mathrm{cm}^{2}$; (b) 2000 pulsos, $\mathrm{D}=2 \mathrm{~mm}$ y $\mathrm{F}_{0}=2.2 \mathrm{~J} / \mathrm{cm}^{2}$.

6. H. Varel, D. Ashkenasi, A. Rosenfeld, M. Wähmer, E.E.B. Campbell: "Micromachining of quartz with ultrashort laser pulses". Appl. Phys. A 65, 367-373 (1997)

7. S. Nolte, C. Momma, H. Jacobs, A. Tünnermann, B.N. Chichkov, B. Wellegehausen, H. Welling: "Ablation of metals by ultrashort laser pulses". J. Opt. Soc. Am. B 14, 2716-2722 (1997)

8. Ver contribuciones en “Laser Ablation, Proc. $5^{\text {th }}$ Int. Conf.", ed. J.S. Horwitz, H.-U. Krebs, K. Murakami, M. Stuke: Appl. Phys. A 69 (Suppl), (1999)

9. H.G. Rubahn: "Laser Applications in Surface Science and Technology". John Wiley \& Sons, Chichester (Reino Unido) 1999.

10. Ver contribuciones en "Laser Precision Microfabrication LPM 2002", ed. K. Sugioka: RIKEN Review 50 (2003)

11. G. Kamlage, T. Bauer, A. Ostendorf, B.N. Chichkov: “Deep drilling of metals by femtosecond laser pulses". Appl. Phys. A 77, 307-310 (2003)

12. N.H. Rizvi: "Femtosecond laser micromachining: current status and applications". RIKEN Review 50, 107-112 (2003)

13. Ver http://www.cmxr.com

14. D. Strickland, G. Mourou: "Compression of amplified chirped optical pulses". Opt. Commun. 56, 219-221 (1985)

15. G. Dumitru, V. Romano, H.P. Weber, M. Sentis, W. Marine: "Femtosecond ablation of ultrahard materials". Appl. Phys. A 74, 729-739 (2002)

16. P.S. Banks, M.D. Feit, A.M. Rubenchik, B.C. Stuart, M.D. Perry: "Material effects in ultra short pulse laser drilling of metals". Appl. Phys. A 69, S377-S380 (1999)

Recibido: 13.09 .03

Aceptado: 22.06 .04 